\title{
Aprendizaje adaptativo basado en competencias y actividades Adaptive learning based on competences and activities
}

\author{
Alberto Real-Fernández, Rafael Molina-Carmona, Faraón Llorens-Largo \\ arf34@alu.ua.es,rmolina@ua.es, faraon.llorens@ua.es \\ Cátedra Santander-UA de Transformación Digital \\ Universidad de Alicante \\ Alicante, España
}

\begin{abstract}
Resumen- Frente al aprendizaje tradicional de talla única, proponemos un modelo de aprendizaje adaptativo basado en las tecnologías de la información, abierto, colaborativo, flexible y escalable. El modelo propuesto tiene como elementos centrales los conceptos de competencia y de actividad de aprendizaje y se estructura en tres elementos principales: el cuadro de mando docente (para el diseño del curso en base a competencias y actividades), el espacio de trabajo del estudiante (en que se realizan las actividades formativas y se mantiene el estado de competencias y actividades) y el motor de selección (encargado de la selección de actividades en función del progreso del estudiante en su aprendizaje). El modelo presentado permite la personalización del contenido, adaptado al nivel de conocimientos de cada usuario y a su progreso, y a través de itinerarios de aprendizaje diferentes elegidos por el propio usuario. Incorpora los conceptos de refresco y de refuerzo y la posibilidad de elegir para dotar a los estudiantes de autonomía.
\end{abstract}

Palabras clave: aprendizaje adaptativo, aprendizaje con tecnología, competencias, actividades de aprendizaje

Abstract- Faced with traditional one-size-fits-all learning, we propose an open, collaborative, flexible and scalable adaptive learning model based on information technologies. The central elements in the model are the concepts of competence and learning activity and it is structured in three main elements: the teaching board (for a course design based on competencies and activities), the student work space (in which the training activities are carried out and the state of competences and activities are maintained) and the selection engine (responsible for the selection of activities according to the student's learning progress). The presented model allows the customization of the content, adapted to the level of knowledge and the progress of each user, and through different learning itineraries chosen by the user. It incorporates the concepts of refreshment, reinforcement and freedom of choice, so that the students are provided with autonomy.

Keywords: adaptive learning, technology-enhanced learning, competences, learning activities

\section{INTRODUCCIÓN}

La fuerte implantación de las Tecnologías de la Información (TI) en nuestra sociedad ha supuesto una revolución que ha alcanzado a todos los ámbitos de la sociedad. En particular, la educación está inmersa en una etapa de convulsión para adecuarse a las necesidades presentes hoy en día, para una transformación digital. Estamos siendo testigos de un cambio en las herramientas empleadas por instituciones y docentes que abren un amplio conjunto de nuevas posibilidades, alternativas a la docencia tradicional (Carneiro, Toscano, \& Díaz, 2009; Llorens Largo, 2009).

La transformación tecnológica no puede llevarnos a una mera actualización de las herramientas, sino que debe englobar todo el modelo y el proceso de enseñanzaaprendizaje. Si no hay una verdadera transformación, las expectativas de una contribución potencial a la mejora del aprendizaje pueden no cumplirse (Punie, Zinnbauer, \& Cabrera, 2006). De hecho, la mayoría de herramientas surgidas bajo el concepto e-learning todavía no aprovechan las capacidades de las TI, replicando el aprendizaje estático y secuencial tradicional pero eliminando la parte de interacción personal tan provechosa en la educación presencial.

La evolución de las TI y la irrupción de internet ha hecho que, a día de hoy, la gran mayoría de las universidades oferten estudios online (Llorens Largo, 2015a). Prácticamente todas ellas disponen de entornos virtuales para gestionar este tipo de aprendizaje (LMS) y muchas de ellas se han lanzado a incorporar plataformas para ofrecer cursos masivos abiertos online (MOOC). Los primeros son gestores de contenidos y recursos de aprendizaje, generalmente de acceso privado, que incorporan herramientas de interacción y comunicación, y en ocasiones de evaluación, entre otras características (Carneiro et al., 2009). Los segundos ofrecen los materiales en abierto y sin restricción de acceso, pero adolecen frecuentemente de falta de universalidad y accesibilidad educativa y una alta tasa media de abandono. En cualquier caso, estas plataformas son rígidas por lo general, con un contenido preestablecido, estructurado de manera predeterminada y uniforme (todos los alumnos se encuentran con exactamente los mismos materiales, sin tener en cuenta sus capacidades o necesidades particulares). Estas experiencias no pueden verse como referentes fracasados, sino que constituyen la base que marca el comienzo de un cambio irreversible en la educación (Delgado Kloos, 2014; Llorens Largo, 2015b).

Fuera del ámbito de las enseñanzas regladas, se han desarrollado plataformas novedosas que persiguen un modelo educativo individualizado, constante y progresivo, que se adapta a las diferentes necesidades o conocimientos del estudiante. Un caso paradigmático es Duolingo, que se ha constituido en un referencia en las aplicaciones de e-learning de idiomas. Este tipo de herramientas incorporan algunas características de aprendizaje realmente adaptativo, aunque siguen siendo experiencias muy centradas en determinados ámbitos y son totalmente cerradas (Real Fernández, 2016). 
Nos encontramos ante la necesidad de preparar a los estudiantes para un aprendizaje y formación continuos, hacerles saber que deben asumir un ciclo continuo de aprendizaje, desaprendizaje y reaprendizaje. Se trata de fomentar en esta nueva generación un aprendizaje autónomo y sujeto a una posible caducidad, algo que en parte han desarrollado, con la mencionada capacidad autodidacta respecto al uso de las nuevas tecnologías. Frente a un concepto de aprendizaje lineal y predefinido, aparece el concepto de aprendizaje adaptativo. Un concepto que, a pesar de estar presente en el mundo de la educación desde hace años, ha empezado a hacerse presente recientemente. Aunque tradicionalmente los profesores han ayudado a los estudiantes según sus capacidades y progresos personalizando el proceso de enseñanza aprendizaje, hoy en día la ayuda de las TI puede permitir un aprendizaje adaptativo real en el que las herramientas educativas se adapten a las necesidades de aprendizaje específicas de cada estudiante (Fleming, 2014).

Cada estudiante tiene habilidades particulares y aprende de una manera diferente, a diferentes ritmos e incluso podría tener diferentes estilos de inteligencia (Gardner, 2000, 2011). La enseñanza de "talla única", procedente de la era industrial y la producción en serie, no es apropiada para el mundo digital (Robinson \& Aronica, 2014). Reigeluth (Reigeluth, 2011, 2012) explica que es necesario definir un nuevo paradigma de formación para la Sociedad de la Información. Su teoría instruccional se centra en el estudiante, y el progreso del estudiante se basa en el aprendizaje en lugar del tiempo. Según Csikszentmihalyi (Csikszentmihalyi, 1990), cuando a un estudiante se le plantea una tarea adecuada a sus habilidades, el estudiante entra en un estado de "flujo", en el que la motivación es máxima. Sin embargo, si la tarea es demasiado difícil se provoca ansiedad, y si es demasiado fácil, se produce aburrimiento. El estudiante, por lo tanto, debe ser mantenido en un estado de flujo, y paso a paso, mientras sus habilidades mejoran, aumentar la dificultad de los retos planteados.

Los actuales avances tecnológicos, concretados en desarrollos en el ámbito de las analíticas de aprendizaje (Siemens, 2012; Villagrá Arnedo et al., 2015), la gamificación aplicada a la educación (Llorens Largo et al., 2016; Prensky, 2006), los sistemas de aprendizaje adaptativos e inteligentes basados en web (Brusilovsky \& Peylo, 2003) y la combinación de estas técnicas (Johnson et al., 2013), nos permiten determinar que las tecnologías actuales están maduras para permitir la creación sistemas de aprendizaje adaptativo, activo, autónomo y persistente. Activo, porque es importante que ese papel recaiga en el estudiante, resultando esenciales en el proceso la motivación y el compromiso del mismo. Autónomo, de modo que el proceso no requiera de una supervisión presencial constante, pueda muchas veces evaluar por sí mismo el progreso de cada alumno y aprender de sus necesidades. Y persistente, porque es importante que lo aprendido lo sea de verdad, es decir, que los conocimientos adquiridos perduren, y el estudiante sea capaz de seguir aprendiendo los nuevos que estén por llegar (Llorens-Largo et al., 2016). En este trabajo presentamos un sistema para la creación de herramientas de aprendizaje adaptativo que pretende integrar todos estos conceptos.

\section{CONTEXTO}

Nuestra propuesta se fundamenta, entre otros aspectos, en la necesidad de mejorar la motivación del estudiante. Esta motivación, que puede ser extrínseca e intrínseca, se encuentra influida por diferentes factores, como se recoge en la llamada Teoría de la Autodeterminación (Ryan \& Deci, 2000). Dicha teoría defiende que, para poder obtener resultados positivos, los elementos empleados en la motivación del usuario no deben basarse únicamente en la motivación extrínseca y deben buscar la motivación intrínseca, en una medida equilibrada.

El objetivo principal de esta investigación es proponer un modelo de aprendizaje adaptativo basado en las TI, abierto, colaborativo, flexible y escalable. Este modelo está orientado a cualquier ámbito educativo, aunque se ilustra con un caso de estudio de un curso de inglés sencillo. Para conseguir este objetivo, proponemos un conjunto de objetivos específicos, inspirados en la Teoría de la Autodeterminación, particularmente en los factores que se utilizan para el diseño de experiencias gamificadas de aprendizaje (Villagrá-Arnedo, Gallego-Durán, Molina-Carmona, \& Llorens-Largo, 2016). Estos objetivos específicos son:

- Diseñar un modelo que permita la personalización del contenido, es decir, que ofrezca un itinerario de aprendizaje diferente según el usuario, ya sea tanto en variedad como en dificultad, adaptado al nivel de conocimiento de cada uno.

- Incorporar los conceptos de refresco (propiciar que no se olviden conceptos ya aprendidos) y de refuerzo (afianzar conceptos a través de actividades alternativas).

- Adaptar el aprendizaje al progreso de forma que el ritmo dependa de la cadencia en la que va superando competencias. El progreso no puede ser homogéneo sino en función de los aciertos y los errores.

- Proporcionar diferentes vías de contenido o distintos itinerarios de aprendizaje, de modo que pueda elegir entre varias opciones para progresar. Esto fomenta la autonomía para que el usuario perciba que es dueño de su proceso de aprendizaje.

- Ofrecer gran variedad de actividades, tanto en la forma en la que se realizan como en el tipo de conocimiento.

\section{DESCRIPCIÓN}

El modelo propuesto tiene como elementos centrales los conceptos de competencia y de actividad de aprendizaje. Una competencia es la combinación de conocimientos, destrezas y actitudes necesarias para una acción eficaz ante un determinado problema. Una actividad de aprendizaje es una acción o tarea que lleva a los estudiantes que la realizan a desarrollar una o varias competencias y, por tanto, a aprender. Los componentes que forman el modelo propuesto son:

- $\quad$ El cuadro de mando docente, que permite el diseño del curso por parte de los docentes e incluye el mapa de competencias y la bolsa de actividades

- $\quad$ El espacio de trabajo del estudiante, en que se realizan las actividades formativas haciendo uso de una instancia del mapa de competencias y de la bolsa de actividades

- El motor de selección que elige las actividades en función del progreso del estudiante en su aprendizaje.

En la figura 1 se representan gráficamente estos elementos y en los apartados siguientes se explican con detalle. 


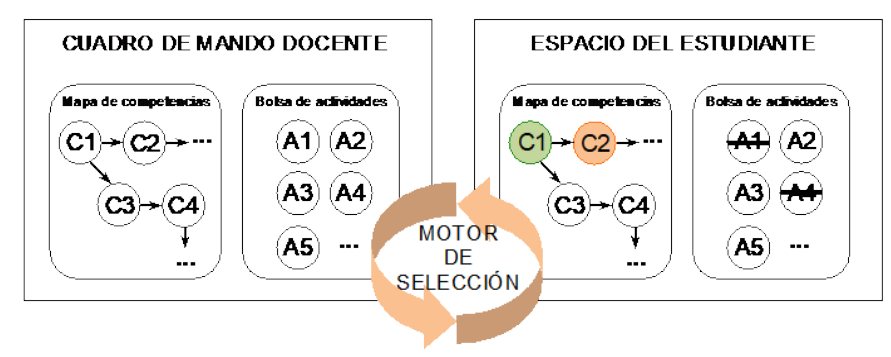

Figura 1: Principales elementos del modelo de aprendizaje adaptativo.

\section{A. El mapa de competencias}

El mapa de competencias es un mapa conceptual que contiene los conocimientos y competencias a desarrollar en el curso en cuestión. Este mapa representa los posibles caminos que podrán seguir los estudiantes a través de todas las competencias disponibles. El mapa de competencias tiene forma de grafo, en el que se incluyen, como principales elementos los nodos y los arcos entre ellos.

Los nodos representan las competencias que debe adquirir cada estudiante. El grafo debe tener uno o más nodos iniciales, que indican el comienzo del camino de aprendizaje del estudiante, es decir, la competencia o competencias por las cuales debe comenzar. Del mismo modo, tiene uno o varios nodos finales, que marcan las competencias en las que debe acabar. Un mapa, y con ello el curso correspondiente, se considerará superado cuando se haya completado un camino desde una competencia inicial hasta una final.

Cada competencia cuenta con dos atributos denominados umbrales de competencia, que indican los niveles mínimo y máximo de la competencia. El umbral mínimo indica el mínimo nivel adquirido en esa competencia para que se considere superada. El umbral máximo indica el nivel máximo a partir del cual, aunque se siga trabajando en esa competencia, no es posible aumentar el nivel. En apartados posteriores se explicarán con detalle estos conceptos.

Los arcos entre los nodos indican relación de precedencia entre las competencias. Los arcos son dirigidos, es decir, para poder adquirir una competencia $\mathrm{C}_{i+1}$ será necesario haber adquirido aquellas competencias desde las que existe un arco (por ejemplo la $C_{i}$ si existe el arco $C_{i} \otimes C_{i+1}$ ). Los arcos también disponen de un atributo denominado umbral de conexión. El umbral de conexión para el arco $\mathrm{C}_{\mathrm{i}} \otimes \mathrm{C}_{\mathrm{i}+1}$ indica el nivel mínimo que debe alcanzarse en la competencia $\mathrm{C}_{i}$ para desbloquear la competencia $\mathrm{C}_{\mathrm{i}+1}$. Además, es posible establecer relaciones múltiples entre competencias a través de conexiones lógicas OR o AND, de modo que en algunos casos bastará con superar cualquiera de las competencias de las que dependa y en otros será necesario superarlas todas.

El docente es el responsable del diseño del mapa, creando todas las competencias que desee para el curso, estableciendo las relaciones de dependencia existentes entre ellas y sus requisitos. Este diseño definirá el modelo de aprendizaje que el docente desee establecer, pudiendo crear desde un modelo básico, a través de un mapa lineal, con unas competencias secuenciales, hasta un modelo complejo, en el que las competencias formarán una red más elaborada.

\section{B. La bolsa de actividades}

La bolsa de actividades contiene todas las actividades disponibles para que sean realizadas por los estudiantes. Este conjunto será creado por los docentes. Las actividades son seleccionadas para ser realizadas por cada estudiante, cubriendo cada actividad una o varias competencias, sirviendo para desbloquear y completar las competencias existentes.

Cada actividad tiene dos atributos principales: las competencias que cubre y su nivel de dificultad. Cuando la actividad se supera con éxito, el nivel alcanzado por el estudiante para esa competencia sube en función de la dificultad: completar con éxito una actividad de una dificultad alta supondrá mayor puntuación que una de dificultad baja.

El docente establece todo el conjunto de actividades del curso. Aunque este diseño de actividades es libre, consideramos que es conveniente que las actividades sean muy variadas. En todo caso, si perseguimos que el sistema esté lo más automatizado posible, debemos tender a construir actividades que puedan desarrollarse on-line, facilitando, en algunos casos, su evaluación automática. Si consiguiéramos que todas las actividades pudieran evaluarse de forma automática, tendremos un modelo totalmente autónomo, que no necesitará de la intervención del docente. Aunque el modelo es independiente de la forma en que se creen las actividades, la filosofía del propio sistema recomienda la creación de actividades que puedan ser compartidas por los docentes, generando así un gran banco de actividades muy amplio que dotarán al sistema de un verdadero aprendizaje adaptativo. Deberíamos considerar también la posibilidad de que los propios estudiantes puedan proponer tipos de actividades diferentes, e incluso crear actividades nuevas. Aprovecharíamos así las teorías constructivistas que abogan porque el estudiante construya su propio aprendizaje de forma activa (Jonassen, 1994; Tryphon \& Vonèche, 2000).

\section{El espacio de trabajo del estudiante}

Una vez diseñado el grafo y la bolsa de actividades, procede poner en marcha el curso. En ese momento, el sistema crea una instancia para cada estudiante de la bolsa de actividades y otra del grafo, asociando también, para ese estudiante, una fuerza o nivel de adquisición de cada competencia.

En el momento inicial todas las conexiones están cerradas y la fuerza de cada competencia se encuentra a 0 . De esta forma, todas las competencias estarán bloqueadas excepto las competencias iniciales. Además, de toda la bolsa de actividades, sólo aquellas asociadas a las competencias iniciales estarán disponibles. En este punto el sistema está en condiciones de proponer al estudiante una actividad de las disponibles. Si el estudiante realiza la actividad con éxito, la puntuación asociada a esa actividad se suma la fuerza de las competencias relacionadas con ella y a los arcos que salen de esas competencias. En este punto se realizan varias acciones:

- Si se supera el umbral de conexión de algún arco, se desbloquean las competencias hacia las que apuntan esos arcos y las actividades que contribuyan a las competencias desbloqueadas (sólo si todas las competencias a las que contribuyen están desbloqueadas).

- Si se supera el umbral mínimo de alguna competencia, ésta se supone superada. Esto indica que en el caso de que todas las competencias necesarias estén superadas el curso ya puede terminar para el estudiante. Sin embargo, si el estudiante sigue con el curso (porque falten otras competencias o porque decida continuar mejorando 
aunque ya lo tenga superado) el sistema puede seguir asignando actividades que contribuyan a esa competencia.

- $\quad$ Si se supera el umbral máximo de alguna competencia, las puntuaciones ya no contribuyen a aumentar la fuerza de la competencia.

De esta manera las competencias se irán habilitando conforme se abran las conexiones correspondientes, es decir, conforme se realicen las actividades asociadas y se superen los diversos umbrales. Igualmente, las actividades asociadas a competencias que hayan sido desbloqueadas pasarán a estar disponibles. A partir de este funcionamiento básico, se pueden establecer mecanismos para introducir el refresco en el sistema. Por ejemplo, disminuyendo con el paso del tiempo la fuerza alcanzada, para forzar a realizar nuevas actividades relacionadas con esa competencia.

La instancia individual del mapa y la bolsa de actividades define el espacio de trabajo de cada estudiante. Este espacio contiene el progreso concreto del estudiante, es decir, el estado de las competencias (bloqueadas o desbloqueadas, el camino recorrido, y el que le queda por recorrer) y de la bolsa de actividades (cuáles están disponibles según las competencias desbloqueadas, y cuáles de ellas ha realizado y cuáles no). En la figura 1 se puede ver el espacio de trabajo de un estudiante, suponiendo un mapa y bolsa de actividades concretos. En la imagen, podemos ver en el mapa el progreso actual del estudiante, donde podemos observar una competencia desbloqueada (en naranja), una completada (en verde), y dos todavía bloqueadas (en blanco). A la derecha, podemos ver que, partiendo de un total de cinco actividades, el estudiante ha realizado dos de ellas (A1 y A4, tachadas).

\section{El motor de selección}

El motor de selección elige en cada comento qué actividad se asigna a cada estudiante. Para ello, debe tener en cuenta:

- El estado de la instancia individual del mapa de competencias, es decir, con qué fuerza se ha desarrollado cada competencia y cuáles están desbloqueadas.

- El estado de la instancia individual de la bolsa de actividades, es decir, qué actividades están desbloqueadas, de todas éstas cuáles han sido ya superadas y las dificultades de las actividades que faltan por realizar.

A partir de ahí, podemos diseñar un algoritmo que teniendo en cuenta estos factores decida cuál es la siguiente actividad que se asigna al estudiante. El diseño de este motor puede ser muy complejo, incluyendo factores como el estilo de aprendizaje del estudiante, su historial en el sistema o sus preferencias, y utilizando modelos computacionales y técnicas de inteligencia artificial muy diversos. Además, el diseño del motor puede ser abierto y compartido, de manera que puede ponerse a disposición de los docentes diferentes motores que realicen la selección de actividades de diferente manera según los objetivos del docente. Como ejemplo, a continuación presentamos un motor sencillo pero que cumple con los requisitos mínimos, y que tiene dos características principales:

- La actividad propuesta no puede haber sido realizada previamente

- Todas las competencias asociadas a la actividad propuesta deben encontrarse desbloqueadas.
Todas las actividades que cumplan estos dos requisitos constituyen un conjunto de actividades candidatas a ser propuestas. A cada actividad candidata se le asigna un valor de prioridad que viene dado por:

- $\quad$ La dificultad de la actividad, en relación con la capacidad del estudiante, es decir, dado el nivel de dificultad media que el estudiante es capaz de superar, se da mayor prioridad a aquellas actividades cuya dificultad se encuentra cercana a ese nivel.

- La fuerza de las competencias que cubra cada actividad, de forma que se da prioridad a aquellas actividades que cubren competencias que están menos desarrolladas (tienen menor fuerza).

Con esto tenemos un conjunto de entrada para el algoritmo que realiza la selección entre las candidatas. La selección puede ser aleatoria entre las candidatas o ponderada por dificultad de la actividad, por número de competencias superadas o por número de dependencias cubiertas.

\section{Resultados}

Una vez explicado el modelo, un caso de estudio nos permite ilustrar su funcionamiento y comprobar si se han cumplido los objetivos. El caso de estudio es un sencillo curso de inglés, de forma simple, con un número pequeño de competencias y posibles actividades. La tabla 1 presenta, codificadas, las 5 competencias del curso.

Tabla 1: Competencias del curso de ejemplo

\begin{tabular}{|c|c|}
\hline Código & Competencia \\
\hline C1 & Present simple \\
\hline C2 & Present continuous \\
\hline C3 & Past simple \\
\hline C4 & Prepositions \\
\hline C5 & Questions \\
\hline
\end{tabular}

La primera acción del docente es disponer las competencias en el mapa, definiendo cuáles son iniciales y finales, qué relaciones de orden se establecen entre ellas, qué umbrales máximo y mínimo se asignan a cada competencia, y qué umbrales de conexión se establecen entre ellas. En el mapa de ejemplo de la figura 2, la competencia C5 tiene una dependencia doble, de C2 y de C3, que en este caso ha sido establecida con una puerta AND, por lo que C5 solo podrá ser desbloqueada cuando las dos competencias superen sus respectivos umbrales de conexión.

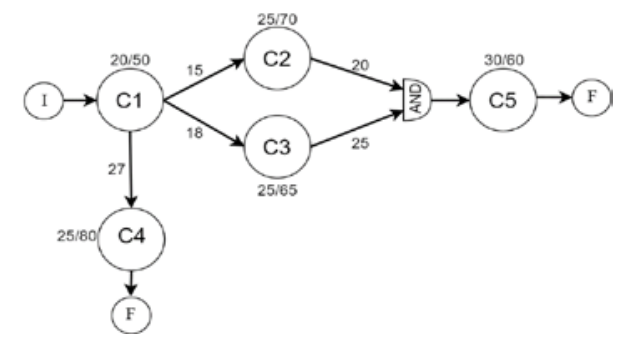

Figura 2: Mapa de competencias con los umbrales de competencia mínimo y máximo, y los umbrales de conexión.

El siguiente paso es la construcción de la bolsa de actividades. Cada actividad propuesta debe venir acompañada de la lista de competencias que cubre y de la dificultad asociada. En la tabla 2 tenemos un ejemplo de bolsa de actividades para nuestro ejemplo con tres posibles valores de dificultad (D1, D2 o D3) y con una puntuación asociada a cada 
una de ellos (en caso de acierto se suman 8, 15 y 25 puntos, y en caso de fallo, se restan 5, 10 y 15 puntos, respectivamente).

Tabla 2: Bolsa de actividades con sus competencias y dificultad

\begin{tabular}{|c|c|c|}
\hline Actividad & Competencias & Dificultad \\
\hline A1 & C1 & D1 \\
\hline A2 & C1, C3 & D1 \\
\hline A3 & C1 & D2 \\
\hline A4 & C1, C3 & D2 \\
\hline A5 & C1, C2 & D1 \\
\hline A6 & C1, C2, C4 & D1 \\
\hline A7 & C2, C4 & D2 \\
\hline A8 & C1, C3 & D2 \\
\hline A9 & C1, C2 & D3 \\
\hline A10 & C1, C2, C3 & D2 \\
\hline A11 & C3 & D1 \\
\hline A12 & C3, C2 & D1 \\
\hline A13 & C3 & D2 \\
\hline A14 & C3, C4 & D1 \\
\hline A15 & C5, C1 & D1 \\
\hline A16 & C5, C4 \\
\hline \multicolumn{2}{|c|}{}
\end{tabular}

Para cada estudiante, el curso se inicia generando una instancia del mapa de competencias con todos los valores de fuerza a 0 y C1 como única competencia desbloqueada por ser la inicial. Se genera también una instancia de la bolsa de actividades, en la que están disponibles las asociadas a la competencia desbloqueada. Las candidatas son A1 y A3. El estudiante selecciona la competencia $\mathrm{C} 1$ y el sistema le asigna, por ejemplo, la actividad A1. Si la realiza correctamente, la fuerza de las competencias asociadas (en este caso sólo la C1) se incrementa (con 8 puntos por ser de dificultad D1), y la actividad se marca como realizada (figura 3). Como ni los umbrales de competencia ni de conexión se alcanzan, no se desbloquean nuevas competencias ni actividades. A3 queda como única actividad disponible y se propone a continuación.

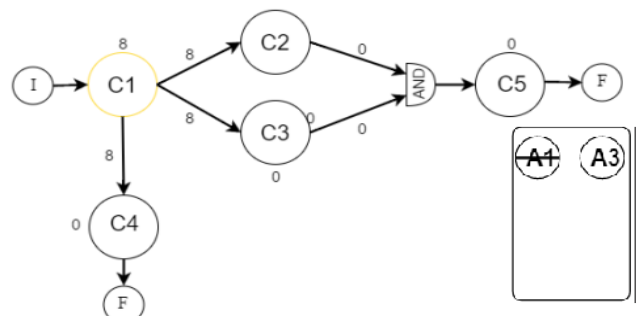

Figura 3: Instancia actualizada del mapa de competencias y de la bolsa de actividades. La competencia C1 está desbloqueada. La actividad A1 se marca como realizada.

Si el estudiante la resuelve con éxito, se marca como completada, y como es de dificultad D2, la fuerza de la competencia C1 aumenta en 15 puntos, sumando un total de 23 y superando el umbral de la competencia (se considera superada) y dos de los umbrales de conexión (se desbloquean C2 y C3 cuyos umbrales eran 15 y 18, respectivamente). La bolsa de actividades incorpora, además, nuevas actividades que están asociadas a las competencias C1, C2 y C3 (figura 4).

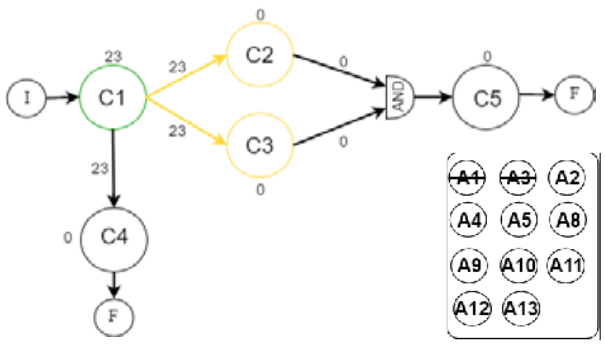

Figura 4: Instancia actualizada del mapa de competencias y de la bolsa de actividades. La actividad C1 está completada (en verde).
Ahora, el estudiante escoge la competencia C2, y el motor de selección le asigna la actividad A10, de dificultad D2. La realiza correctamente, y actualizamos la fuerza de las competencias que cubre la actividad A10 (C1, C2 y C3). Las fuerzas de las competencias aumentan en 15 puntos, se supera umbral de $\mathrm{C} 1 \otimes \mathrm{X} 4$ (C4 pasa a estar desbloqueada), y se añaden las actividades A6, A7 y A14 a la bolsa (figura 5).

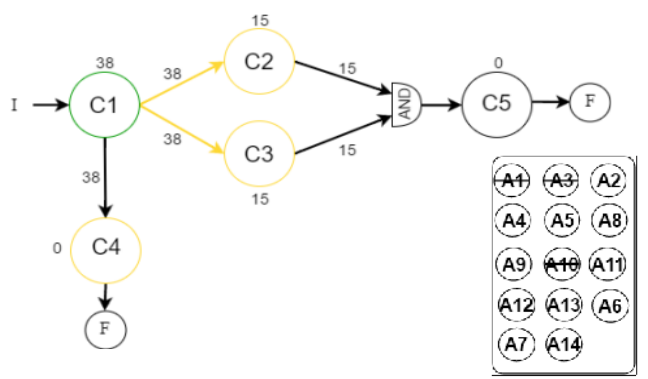

Figura 5: Instancia actualizada del mapa de competencias y de la bolsa de actividades.

Cuando el estudiante no realiza una actividad correctamente, el sistema resta los puntos correspondientes en los valores de fuerza de competencia y de conexión asociados a dicha actividad. El proceso continúa hasta que el estudiante alcanza alguna competencia final habiendo superado todos los umbrales de las competencias que hay en el camino entre una competencia inicial y otra final.

\section{CONCLUSIONES}

Hemos descrito un modelo de aprendizaje adaptativo, basado en las TI, abierto, colaborativo, flexible y escalable. Es abierto y colaborativo puesto que permite la participación de cualquier docente y la compartición de sus actividades y estrategias; es flexible puesto que permite que el docente elabore su curso con total libertad y es escalable, puesto que el sistema es independiente de la complejidad del curso y del número de competencias y de actividades. En particular, se cumplen los objetivos:

- Permite la personalización del contenido, al ofrecer la posibilidad de diferentes itinerarios de aprendizaje, con toda la variedad de actividades y diferentes niveles de dificultad que elija el docente.

- Los errores restan puntos, por lo tanto, fallar obliga a recuperar los puntos con nuevas actividades, es decir, se propicia el refuerzo de los conceptos menos afianzados. Además, la disponibilidad de actividades variadas permitirá que se refuercen esas competencias realizando actividades diferentes. El concepto de refresco puede incorporarse también haciendo que el paso del tiempo sin realizar actividades asociadas a una competencia suponga una pérdida de fuerza que obligue a refrescarla con nuevas actividades.

- El motor de selección asigna diferentes actividades, teniendo en cuenta el estado de la instancia del mapa y de la bolsa de actividades, así como la dificultad de las actividades y la destreza del estudiante. En definitiva, el aprendizaje se adapta al ritmo y características del estudiante, haciendo que el progreso se adapte (estado de flujo). 
- $\quad$ Se pueden establecer diferentes competencias iniciales y finales, introducir operadores lógicos... En definitiva, puede haber distintos itinerarios de aprendizaje, de modo que se puede elegir entre varias opciones para progresar.

En el futuro nos proponemos avanzar en el desarrollo de este modelo y en la construcción de un prototipo que permita validar el funcionamiento. A nivel práctico, se estudiará la integración del modelo con LMS existentes. Por otro lado, puesto que uno de los elementos centrales del aprendizaje es la actividad, el esfuerzo se centrará en el diseño y construcción de estas actividades. El otro elemento central es el aprendiz, para el que estudiaremos la forma de definir un modelo de usuario, que incluya cuestiones como los estilos de aprendizaje y de pensamiento, y que pueda integrarse en el motor de selección de actividades.

\section{REFERENCIAS}

Brusilovsky, P., \& Peylo, C. (2003). Adaptive and Intelligent Web-based Educational Systems. Int. J. Artif. Intell. Ed., 13(2-4), 159-172.

Carneiro, R., Toscano, J. C., \& Díaz, T. (2009). Los desafíos de las TIC para el cambio educativo. Madrid, España: OEI : Fundación Santillana.

Csikszentmihalyi, M. (1990). Flow: The Psychology of Optimal Experience. Harper \& Row.

Delgado Kloos, C. (2014, November). En Busca de la Fórmula Mágica en Educación. Conferencia Invitada presented at the XVI Simposio Internacional de Informática Educativa, SIIE 2014, Logroño (Spain).

Fleming, B. (2014, April 1). Adaptive Learning Technology: What it is, Why it matters. Retrieved May 16, 2017, from http://www.eduventures.com/2014/04/adaptivelearning-technology-matters/

Gardner, H. (2000). Intelligence reframed: multiple intelligences for the 21st century. New York, NY: Basic Books.

Gardner, H. (2011). Multiple Intelligences: Reflections After Thirty Years. National Association of Gifted Children Parent and Community Network Newsletter. Retrieved from

https://howardgardner01.files.wordpress.com/2016/04/4 72-multiple-intelligences-reflections-after-30-years.pdf

Johnson, L., Adams, S., Cummins, M., Estrada, V., Freeman, A., \& Ludgate, H. (2013). The NMC Horizon Report: 2013 Higher Education Edition. New Media Consortium. Retrieved from http://www.editlib.org/p/46484

Jonassen, D. H. (1994). Thinking Technology: Toward a Constructivist Design Model. Educational Technology, 34(4), 34-37.

Llorens Largo, F. (2009). La tecnología como motor de la innovación educativa. Estrategia y política institucional de la Universidad de Alicante. Arbor, CLXXXV(Extra), 21-32. https://doi.org/10.3989/arbor.2009.extran1203

Llorens Largo, F. (2015a). Campus virtuales: de gestores de contenidos a gestores de metodologías. Revista de Educación a Distancia, 0(42).
Llorens Largo, F. (2015b). Dicen por ahí ... que los MOOC han muerto. ReVisión (Revista de Investigación En Docencia Universitaria de La Informática), 10(1).

Llorens Largo, F., Gallego Durán, F. J., Villagra Arnedo, C. J., Compan-Rosique, P., Satorre Cuerda, R., \& MolinaCarmona, R. (2016). Gamificación del Proceso de Aprendizaje: Lecciones Aprendidas. VAEP-RITA, 4(1), 25-32.

Llorens-Largo, F., Villagrá-Arnedo, C. J., Gallego-Durán, F. J., Satorre-Cuerda, R., Compañ-Rosique, P., \& MolinaCarmona, R. (2016). LudifyME. In Formative Assessment, Learning Data Analytics and Gamification (pp. 245-269). Elsevier.

Prensky, M. (2006). Don't bother me mom-I'm learning! Paragon House Publishers.

Punie, Y., Zinnbauer, D., \& Cabrera, M. (2006). A Review of the Impact of ICT on Learning (Technical Note No. JRC 47246) (p. 24). Sevilla (Spain): European Commission Joint Research Centre - Institute for Prospective Technological Studies.

Real Fernández, A. (2016). Esbozo para la transformación digital del mundo educativo. Alicante, Spain: Universidad de Alicante. Retrieved from http://hdl.handle.net/10045/58426

Reigeluth, C. M. (2011). An instructional theory for the postindustrial age. Educational Technology Magazine, 51(5), 25-29.

Reigeluth, C. M. (2012). Instructional Theory and Technology for the New Paradigm of Education. Revista de Educación a Distancia, (32).

Robinson, K., \& Aronica, L. (2014). Finding your element: how to discover your talents and passions and transform your life.

Ryan, R. M., \& Deci, E. L. (2000). Self-determination theory and the facilitation of intrinsic motivation, social development, and well-being. The American Psychologist, 55(1), 68-78.

Siemens, G. (2012). Learning analytics: envisioning a research discipline and a domain of practice. In In: International Conference on Learning Analytics and Knowledge (LAK (pp. 4-8). ACM.

Tryphon, A., \& Vonèche, J. J. (2000). Piaget-Vygotsky: la génesis social del pensamiento. Buenos Aires: Paidós.

Villagrá Arnedo, C., Gallego-Durán, F. J., Llorens Largo, F., Compañ, P., Satorre Cuerda, R., \& Molina-Carmona, R. (2015). Detección precoz de dificultades en el aprendizaje. Herramienta para la predicción del rendimiento de los estudiantes. In $\mathrm{La}$ Sociedad del Aprendizaje. Actas del III Congreso Internacional sobre Aprendizaje, Innovación y Competitividad. CINAIC 2015. Madrid, Spain.

Villagrá-Arnedo, C., Gallego-Durán, F. J., Molina-Carmona, R., \& Llorens-Largo, F. (2016). PLMan: Towards a Gamified Learning System. In P. Zaphiris \& A. Ioannou (Eds.), Learning and Collaboration Technologies (Vol. 9753, pp. 82-93). Springer International Publishing. 